\title{
The Heart and the Kidneys: Partners in Disease?
}

\author{
James R. Sowers ${ }^{\mathrm{a}-\mathrm{d}}$ \\ aDivision of Endocrinology, Diabetes and Metabolism, Department of Internal Medicine, \\ ${ }^{b}$ Department of Medical Pharmacology and Physiology, University of Missouri-Columbia \\ School of Medicine, ' Diabetes and Cardiovascular Center, and ${ }^{\mathrm{d}}$ Harry S. Truman VA Medical \\ Center, Columbia, Mo., USA
}

During the past decade, there has been increasing evidence that a complex interplay exists between heart and kidney disease. An important emerging factor that contributes to the epidemic of heart failure and chronic kidney disease is obesity. Currently, more than half of all the adults in the United States, the United Kingdom and other countries in Europe are either overweight or obese, and the rates of overweight and obesity are increasing in all age groups, including children. Excess adiposity is an independent risk factor of all-cause and cardiovascular mortality, the relative risk associated with excess weight being especially pronounced in younger individuals. Overweight and obesity contribute to the increasing prevalence of heart failure, especially that characterized by impaired diastolic function. There is also increasing evidence that excessive fat mass contributes to the development and progression of chronic kidney disease independent of hypertension and diabetes mellitus. There are a number of candidate factors that may link the emerging epidemics of obesity, heart and kidney disease. Indeed, excess adiposity is often associated with metabolic abnormalities, which include insulin resistance, elevated levels of triglycerides and small dense low-density lipoprotein particles, low levels of high-density lipoprotein, elevated glucose and uric acid levels, high blood pressure and vascular endothelial dysfunction. These metabolic abnormalities are often present at an early age, long before clinical manifestations of cardiovascular and chronic kidney diseases become apparent.

Our new journal entitled CardioRenal Medicine was launched to publish results of basic, clinical and epidemiological science that explores the emerging common pathophysiological mechanisms linking heart and kidney disease. This new journal should fill a void, as extant journals typically focus on cardiovascular or kidney disease or factors such as hypertension that promote both cardiovascular and chronic kidney disease. In this context, the first article in the journal focuses on the interplay of factors that contribute to the genesis of the 


\section{CardioRenal \\ Medicine}

cardiorenal syndrome. In this article, emphasis is placed on the role of overweight and obesity in the development of this syndrome and associated heart and kidney disease. The second article in this inaugural edition explores the historical understanding of the linkage between heart and kidney disease, and acknowledges the use of the term cardiorenal syndrome by Guyton and colleagues. The third article explores the use of a prototypic model of obesity and insulin resistance in the investigation of common metabolic factors that contribute to disease in both organs. The remaining articles represent original basic and clinical science research articles that presage research that will be published in future contributions to the journal. To that end, we have an outstanding editorial board that will play a very important role in the future success of CardioRenal Medicine. We also have an outstanding assistant editor, Brenda Hunter, and production editor, Angela Hefti. Finally, we would like to thank Peter Roth and George Bakris for their support and encouragement in initiating this new journal. 